\title{
Advantage of in Service Condition Based Assessment for Transformers in enhancing the maintenance strategy
}

\author{
Ibrahim Al Balushi ${ }^{1}$ \\ ${ }^{1}$ Oman Electricity Transmission Company, Muscat, Sultanate of Oman \\ E-mail:Ibrahim.albalushi@omangrid.com
}

Abstract - controlling the maintenance OPEX is one of the major challenges that any utility faces. The challenges lie in how to optimize the three main factors: risk, performance, and cost. Besides, no utility can depend on a unique type of maintenance, there is always a combination of a different kind of maintenance such as breakdown, preventive, risk-based, conditionbased,..etc. So, what is the answer to this question: what type of maintenance needs to be followed to keep the transformer in service in with high performance? There is no specific answer to this question. Each type of maintenance can be applied based on the transformer's operating environment. However, most of the utilities apply preventive and condition-based maintenance. To justify this answer, some data need to be analyzed to assess the maintenance performance and recommend what are enhancement need to be added. One of these approaches is to apply in service condition-based assessment to study the health of the assets based on the current maintenance practice. Furthermore, study both historical maintenance records and failure rates will help to understand the relationship between the effectiveness of maintenance and service efficiency. This relation can come in two shapes. One is to do the right things by developing a set of maintenance activities that need to be performed during the maintenance to ensure its effectiveness. Second, is to do things right by enhancing the maintenance crew capabilities and competencies to ensure high efficiency. After analyzing all these factors mentioned above, It has been noticed that in-service condition-based assessment of the transformer is a powerful tool that can be used to enhance and build an effective strategy. It will not only involve a set of activities during the maintenance, but it also covers the whole life cycle of the transformer. Besides, it highlights the gaps in the maintenance process and procedures, and provide indications where enhancement need to be applied based on international practice. These changes were observed on the cost and performance in the benchmarking study that was done through International Transmission Operation and Maintenance Study (ITOMS) which was a good indication of the effectiveness of strategy used for transformers. However, as part of the asset management approach, continuous improvement will continue to reach the vision that has been set in the maintenance optimization and to prepare for the future significant increase in transformer aging.

\section{INTRODUCTION}

Asset Management is the science, and art of optimal allocation of resources in the pursuit of achieving given objectives. These objectives may include meeting threshold criteria related to reliability, performance, costs, and prevailing legal mandates. Simply put, the question boils down to, "Where do I invest to achieve the desired objectives, subject to various constraints?" From the Utility's perspective, Asset Management comprises the entire life cycle - cradle to grave - of equipment. Asset Management typically begins with the process of planning and continues through procurement, installation, commissioning, ongoing operation \& maintenance, and salvage. While the first step - planning - addresses the capital allocation issue, the last step - salvaging - lays the foundation for the next round of planning, and capital allocation, thus perpetuating the continuous cycle of Asset Management. Although there were no major transformers failures and it shows a high service performance during the past years, the participation in the international benchmarking showed that transformer OPEX is higher than most of the international transmission utility participating in the study. Besides, increase in the number of the transformer evoked some questions such as how many resources are needed to face these numbers of transformer, how instance can be covered based on the current maintenance practice, are the company willing to accept these increase in the OPEX especially what the decrease in the oil price and the desire from the shareholders to decrease the OPEX. Although the vision was clear, there were many challenges were faced. Any changes in the current maintenance practice need to be supported with data analysis and develop risk assessments associated with these changes. Furthermore, the strategy shall focus on the effectiveness procedure shall concentrate on efficiency. Among several options, the decision was taken study the outcome of the in-service condition assessment that was performed in 2010 and 2015 along with the maintenance history and failure rate to develop a strategy that suite OETC system and monitor its effectiveness though continues the analysis of the reports.

\section{HISTORICAL MAINTENANCE PRACTICE.}

During 2008 and 2018, the population of the transformers has been increased dramatically by the minimums percentage increase of $8 \%$ and the maximum increase $26 \%$ annually. In 2008, the network consisted of both 132 and $220 \mathrm{kV}$ whereas the $400 \mathrm{kV}$ has been introduced in 2016. This rapid increase in the transformer investment met a major challenge in the maintenance according to the maintenance since 2008. As shown in both figures 1 and 2, the maintenance of every transformer consumes 8 hours on average. The previous maintenance practice involved some of the basic activities such as cleaning, measure the isolation resistance of the winding, operation test...etc. From 2008 to 2015 and due to the increase in the number of transformers, the maintenance crew faced challenges to treat all 
transformers in a limited duration from October to April. As shown in figure 3, the maintenance duration involves 1768 hours. It is noticed that the maintenance hours for 2017 and 2018 were higher than allowed hours. Although there is more than one maintenance team that can perform the maintenance, it is worth to mention that this maintenance period is for maintenance activities such as switchgear, axillary system..etc. Besides, the highest priority in this period was given for the projects. With these activities, it is essential to maintain system security, high reliability, and availability. Furthermore, the benchmarking study shows that OETC is spending high costs on the transformer maintenance as it will be shown later. This led to a decision from the management to reduce the cost of the maintenance and find an effective solution and plan to optimize the maintenance. Another factor was considered also as one of the challenges, the number of grid station investments has been increased since 2012. Besides their involvement in the maintenance activities, site engineers are also involved in the commissioning and testing of new projects.

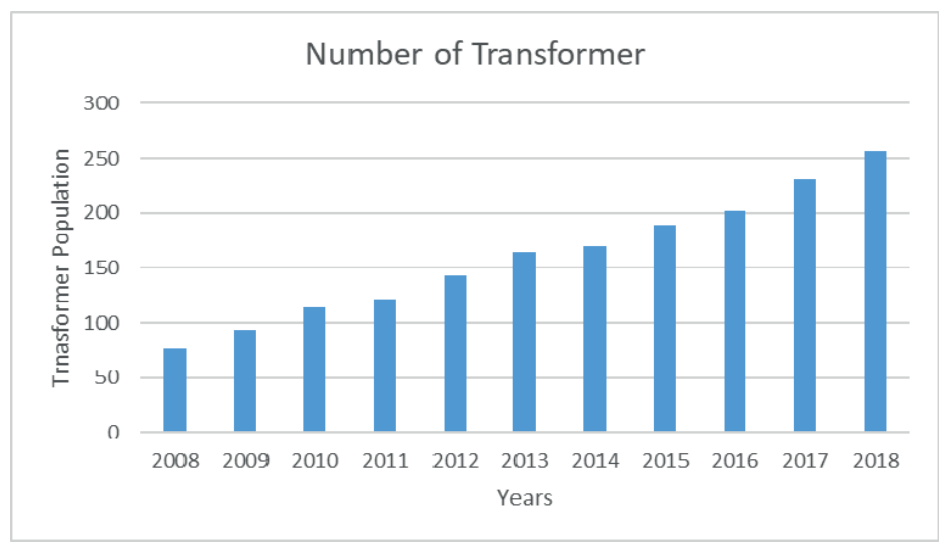

Figure 1: Transformer population 2008-2018

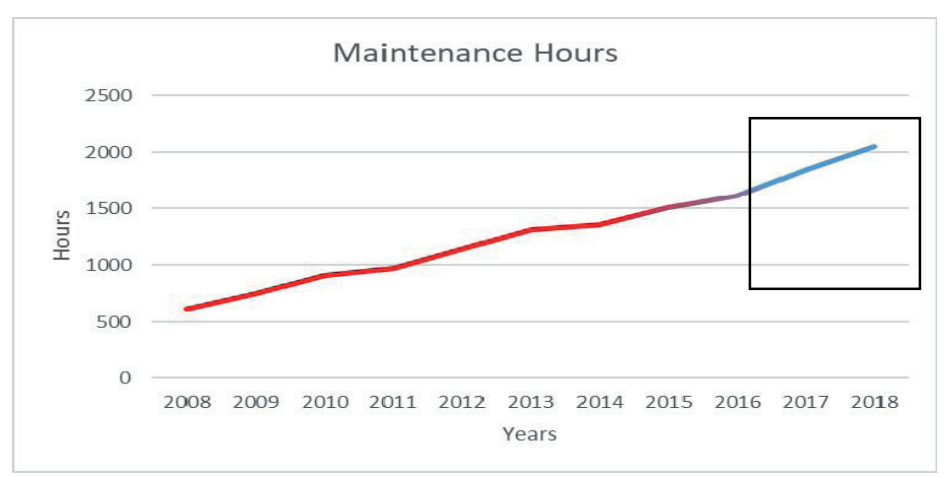

Figure 2: Time spent in the maintenance

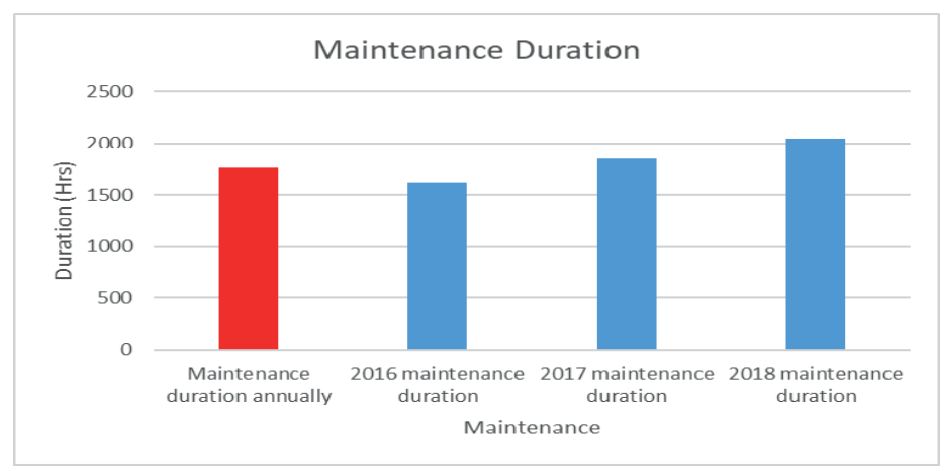

Figure 3: Expected maintenance duration for 2016,2017 \& 2018

\section{CONDITION ASSESSMENT}

To assess achieving the asset management objectives, condition assessment and site tests have been conducted for transformers in the network in both 2010 and 2015. This initiative was a new experience for OETC which was not be used in the past. The condition assessment involves partial discharge, thermal imaging, and visual inspection. Based on 
these factors, the transformer's condition will be categorized according to the maintenance requirements and fast response which will be represented in the form of risk. Despite the availability of the test reports and information from the time-based maintenance, the in-service condition assessment will provide the utility enough information about the status and the condition of its assets while they are under operation. These data can be used to build an asset health index, failure mode, develop an effective asset register, life cycle replacement. However, the main scope of the asset condition assessment will involve the following

- Collection and analysis of relevant (historical) data.

- Detailed Visual Inspection of the Grid Stations and individual assets.

- In service Partial Discharge testing of Transformers using UHF.

- Infrared Thermal Imaging of the Transformers

- Evaluation of test results, identify the weak points in system and suggestion of remedial measures through approved technical reports.

- Health Index ranking for all assets.

Once all data analysis is done, the asset will be ranked based on the criticality of each asset as mentioned in the table below:

Table 1 : risk classifications of the condition assessment findings.

\begin{tabular}{|l|c|}
\hline Risk & Action \\
\hline High & Immediate action \\
\hline Moderate & Within 6-12 months \\
\hline Low & Within 1-5 years \\
\hline Normal & No action is required \\
\hline
\end{tabular}

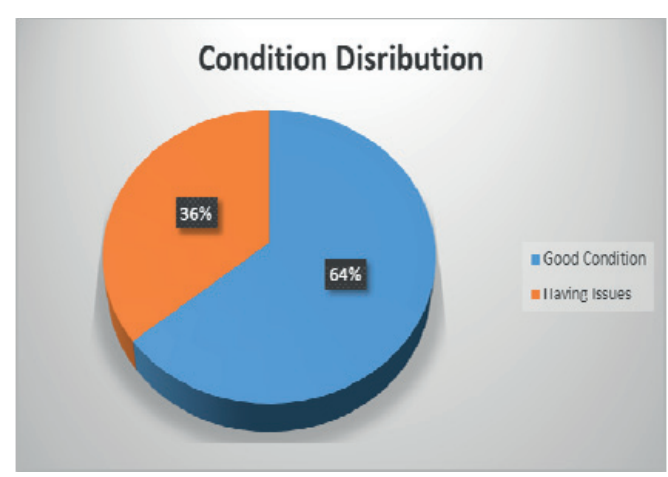

Figure 4: Transformers Condition Distribution in 2010 and 2015

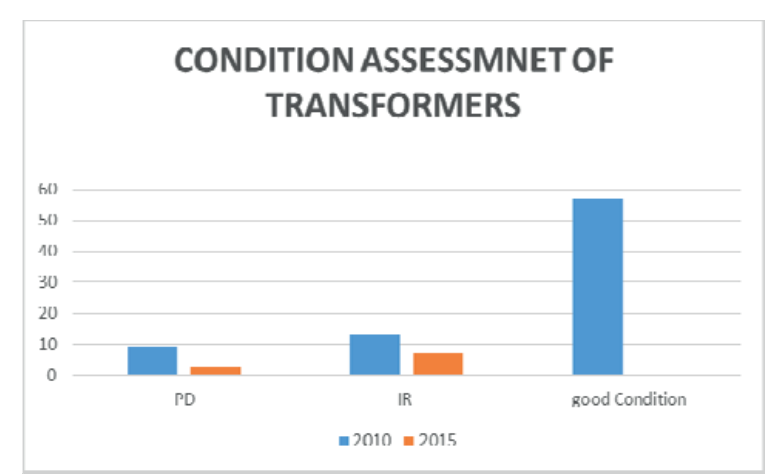

Figure 5: Type of Transformers Findings issues in 2010 and 2015 


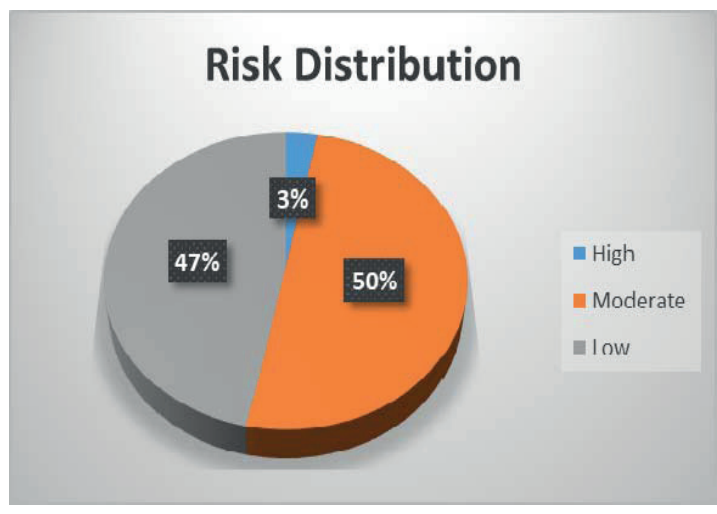

Figure 6: Transformers Risk Distribution 2010 and 2015

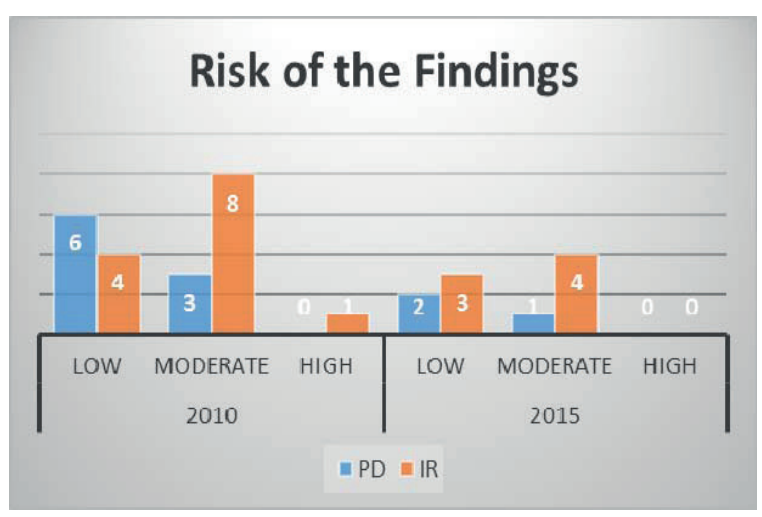

Figure 7: Risk Findings in 2010 and 2015

\section{DATA ANALYSIS.}

Figures 4 \& 5 illustrate the condition assessment of 89 transformers 2010 and 2015. Figure 4 shows the results of 57 transformers sustain good conditions in both years. This result represents $64 \%$ of the total transformers as shown in figure 5. The condition assessment of the Partial Discharge (PD) measurement resultant that there were 9 transformers had PD in 2010. On the other hand, these results encountered a sharp reduction in the number of PD findings in 2015 by around $75 \%$. One of the main observations that had been noticed, none of the 12 transformers had a PD in both years. It can be also seen from figure 4, the results of Infrared (IR) thermography for 20 transformers who got some issues using this kind of test in both years. in 2010, 13 transformers got issues as a result of the IR. Similar to PD measurement, in 2015 there was a decrease in the number of issues found in the transformers compared with 2010 by around $50 \%$. Unlike PD, four transformers got issues in both years using the IR test. Generally, among 89 transformers there were tested in both years, the condition assessment results showed that 32 transformers were found having some issues varied between PD and IR findings. this number represented $36 \%$ of the total tested transformer in both years as shown in figure 5. However, although the findings formed $36 \%$ of the total transformer, these findings have a different range in the risk classifications as shown in table 1. Both Figures 6 and 7 gave distributed condition assessment findings in terms of the risk classifications. $47 \%$ of the transformers have a low risk which means that this risk needs to be cleared within $1-5$ years. in contrast, $50 \%$ of the transformers have a moderate risk. Whereas only $3 \%$ of the transformers lay within the high risk. Only 7 transformers at low risk using IR in both years. There were only a few observations found such as hotspots in one of the transformer parts and some of them related to radiator valves. Some of the valves were found closed which prevent the oil circulation. When the oil circulates, it helps in the transformer cooling by dissipating the heat while the oil circulation. The closing valve will contribute to increasing the heat in some of the parts of the transformer which will be detected by IR. Similarly, 12 transformers were considered to be at moderate risk using the IR test in both years. Most of the observations were related to the transformer oil. The majority of the 12 transformers have some thermal activities which they are not be considered as a moderate. Serval Dissolve gas analysis was conducted to monitor and confirm the condition of these transformers. The results showed that these transformers were subjected to high load and high temperatures during the summer period. As a result, temporary thermal activities occurred for a short period. It is noticeable the only high risk has occurred in 63 MVA transformer which was commission in 1995. It was observed during IR testing that high hotspots in the connection point of the transformer bushing with the conductor. There are two reasons to explain this issue. One of the reasons is due to high resistance, the other reasons can be loose in the connection. This is considered as high risk because it may lead to a short circuit and it could cause severe damage to the transformer itself. On the other hand, PD measurement results are considered to be $37.5 \%$ of the total findings of the condition assessment. The majority of these findings have low risk. Besides, there were only 4 transformers have a moderate risk. A remarkable observation that there were no PD activities were considered to be high risk. PD test results can be classified into two categorize based on the location. Most of the PD detection results were found inside the transformer tank. There was no confirmation regarding the PD source which is can be in the bushing, coil or winding. Sometimes the PD source can be due to a particle /impurities that moves during the oil circulation. This can be confirmed during the Dissolve gas analysis and monitor the trends of some of the key gases within the transformer. PD activities also detected inside the transformer cable box. This is can be due to several reasons such as loose in the connection with bushing, bad insulation condition of the cable or it can be due to low insulation of the substance that OETC used in some of the transformers such as oil or compounds. Although there were no major findings are considered as high risk in the PD results, they need to be taken into consideration and need a contingency plan and continuous monitoring until the clearing the defect. The reason behind this action is that most of 
the transformer fire is generated from the cable box due to PD or corona activities which may develop and their temperature reaches the degree of ignition.

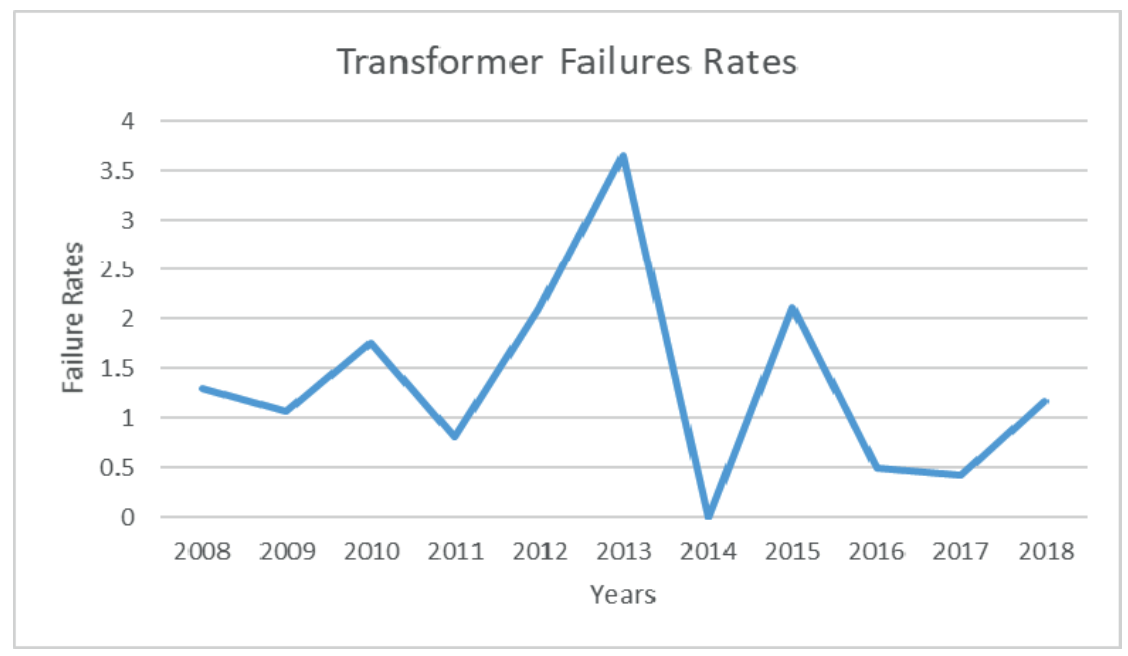

Figure 8: Transformer failure rate

It is essential to study the failure rate of the transformers because that will support the decision making to develop a consistent transformer maintenance strategy. Figure 8 shows the failure rate of the transformer from 2008 to 2018 . it is important to mention that the failure in this paper is defined as a failure to deliver the power due to fault or forced outages. During these years, there were only two major failures were recorded in 2009 and 2011 which required a transformer to be replaced. Other than these two, the failures were considered minor such as overloading, mechanical protection malfunction..etc. it is noticed from the figure that the maximum failure reached 3.6 in 2013 and in 2014 there was no failure recorded. However, these records a simple fact which are maintaining the transformer annually is not required.

\section{CONCLUSION AND RECOMMENDATION}

The in-service condition assessment is an effective tool that can be used to enhance and improve the maintenance strategy. Despite it provides good data about the asset status during operation, offline testing results are essential to building a strong decision about the asset condition. Although there were some observations were considered as high risk, it is considered as individual but not as network risk. Besides, these defects can be cleared within the mentioned plan. The above condition assessment observations that there were some gaps and weaknesses in the maintenance. Furthermore, there are also over or under maintenance frequency in some assets. However, to enhance the maintenance strategy for both Transformers as listed below:

1. Strong need for raising the skill levels for maintenance staff and improve the maintenance process and procedures.

2. Review and improve the current maintenance practice program and procedures against the best practice that suits the OETC network.

3. Retrain maintenance crew and instruct service provider.

4. Enhance the visual inspection of transformers to be every two months.

5. Develop Health and Risk indices for both assets.

6. Develop life cycle replacement.

7. Improve the failure mode of the assets based on the condition assessment which will help to predict when will be the next maintenance/replacement stage.

8. One of the issues that OETC is facing is the overstock of the spare parts. This means that there cost for procurement, storing, auditing, managing and disposing of these spare parts. Building a health and risk indices in line with both failure mode and replacement wave will enhance the spare part management.

9. Enhance the standard specifications and design of the assets.

10. Strengthen the asset register database which will help in both short and long term planning.

11. Increase the general awareness of the risk of improper maintenance.

\section{REFERENCES}

[1] Moubray.J., 1992. Reliability Centered Maintenance. 2th. USA: Industrial Press Inc.

[2] Cigre Guid 248 Power Transformer Failure Rate change by age of unit. 